\title{
Multidrug-resistant surgical site infections in a humanitarian surgery project
}

\author{
R. A. MURPHY ${ }^{1}$, O. OKOLI ${ }^{2}$, I. ESSIEN ${ }^{2}$, C. TEICHER ${ }^{5}$, G. ELDER $^{4}$, \\ J. PENA ${ }^{4}$, J.-B. RONAT ${ }^{4}$ AND K. J. BERNABE ${ }^{3}$ \\ ${ }^{1}$ Division of Infectious Diseases, Los Angeles Biomedical Research Institute at Harbor-UCLA Medical Center, \\ Torrance, CA, USA \\ ${ }^{2}$ Doctors Without Borders, Abuja, Nigeria \\ ${ }^{3}$ Doctors Without Borders, New York, NY, USA \\ ${ }^{4}$ Médecins Sans Frontières, Paris, France \\ ${ }^{5}$ Epicentre, Paris, France
}

Received 29 March 2016; Final revision 14 July 2016; Accepted 15 July 2016; first published online 11 August 2016

\section{SUMMARY}

The epidemiology of surgical site infections (SSIs) in surgical programmes in sub-Saharan Africa is inadequately described. We reviewed deep and organ-space SSIs occurring within a trauma project that had a high-quality microbiology partnership and active follow-up. Included patients underwent orthopaedic surgery in Teme Hospital (Port Harcourt, Nigeria) for trauma and subsequently developed a SSI requiring debridement and microbiological sampling. Data were collected from structured chart reviews and programmatic databases for 103 patients with suspected SSI $[79 \%$ male, median age 30 years, interquartile range (IQR) 24-37]. SSIs were commonly detected post-discharge with $58 \%$ presenting $>28$ days after surgery. The most common pathogens were: Staphylococcus aureus $(34 \%)$, Pseudomonas aeruginosa (16\%) and Enterobacter cloacae $(11 \%)$. Thirty-three $(32 \%)$ of infections were caused by a multidrug-resistant (MDR) pathogen, including 15 patients with methicillin-resistant $S$. aureus. Antibiotics were initiated empirically for $43 \%$ of patients and after culture and sensitivity report in $32 \%$. The median number of additional surgeries performed in patients with SSI was 5 (IQR 2-6), one patient died (1\%), and amputation was performed or recommended in three patients. Our findings suggest the need for active long-term monitoring of SSIs, particularly those associated with MDR organisms, resulting in increased costs for readmission surgery and treatment with late-generation antibiotics.

Key words: Multidrug resistance, nosocomial infection, resource-limited setting, surgical site infection.

\section{INTRODUCTION}

Healthcare-associated infections (HAIs) represent a threat to patient safety globally. The burden of HAI is

\footnotetext{
* Author for correspondence: R. A. Murphy, MD, MPH, Division of Infectious Diseases, Harbor-UCLA Medical Center, 1000 W. Carson Street, Box 466, Torrance, CA, USA. (Email: ramurphy@gmail.com)
}

greater in resource-limited settings where the estimated prevalence is estimated to be $15 \cdot 5 / 100$ patients, exceeding the prevalence reported for Europe at $7 \cdot 1 / 100$ patients and the United States at $4 \cdot 5 / 100$ patients [1]. Nevertheless, the burden of HAIs may be underestimated in low-income countries because of the currently low capacity to identify these events in such settings in the absence of routine hospital surveillance for HAIs. 
Surgical site infection (SSI) is an important category of HAIs with a substantial impact on patient morbidity and mortality. Studies suggest that SSI rates are also far higher in sub-Saharan Africa. A recent prospective study in Kenya documented SSI in $8 \%$ of surgical patients but other estimates have been higher, including an SSI rate of $22 \%$ reported at a rural Tanzanian hospital [2,3]. Despite these estimates, studies that describe SSIs in sub-Saharan Africa that include rigorous microbiological sampling and patient outcomes remain scarce. The development of a linkage between an existing Médecins Sans Frontières (MSF) surgical project in Port Harcourt, Nigeria and a high-quality microbiology facility (Pathcare, Lagos, Nigeria) provided an opportunity to describe serious SSIs requiring operative debridement after orthopaedic trauma in Nigerian patients.

\section{METHODS}

\section{Study population}

Teme Hospital in Port Harcourt, Nigeria - a 70-bed trauma centre - provides emergency medical and surgical care for patients with trauma, most commonly from gunshot wounds, stabbings, road traffic accidents, and sexual violence. MSF's surgical programme at Teme Hospital closed in August 2013. During the study period, from 1 January 2011 to 31 December 2012, about 340 operations were carried out monthly [4, 5] giving a total of 3038 surgical interventions at Teme Hospital in 2011 [6].

The prevention of SSIs was considered a priority at the hospital. To minimize SSI incidence several components were in place including an infection control committee, an infection prevention nurse, and regular visits from technical experts at headquarters. Practice protocols aimed at reducing hospital-acquired infection included guidance on patient selection (e.g. internal fixation was not performed in patients with Type II and III open fractures), skin preparation, and antibiotic prophylaxis. For patients with open fractures, antibiotic prophylaxis was administered in the emergency department immediately upon presentation and consisted of $48-72 \mathrm{~h}$ of broad-spectrum antibiotic therapy; tetanus toxoid was also routinely administered. For closed fractures, a single dose of parenteral cefazolin 30-60 min prior to incision was given. Pre-operative hair removal - when necessary was achieved without the use of a razor and surgical site antisepsis was achieved with $10 \%$ povidone-iodine solution. Operating room equipment was sterilized in a Matachana (Barcelona, Spain) sterilizer with an active quality control of the process.

After initial wash-out and debridement, to achieve wound closure in open fractures, patients underwent repeated wound debridement, received povidone-iodine dressings (if the wound bed was highly contaminated) or wet-to-dry dressings, and, in some cases, split-thickness skin grafts or flap coverage. Nonetheless, infections were common particularly in patients with open fractures, including multidrug-resistant (MDR) pathogens. Space sufficient to individually isolate patients with MDR organisms did not exist at Teme Hospital; therefore a pragmatic approach was employed consisting of (1) cohort isolation of patients with clinical infection in a single ward, (2) an emphasis on hand hygiene with easy accessibility of alcohol-based hand rub and latex gloves, (3) maintenance of a minimal $2 \mathrm{~m}$ distance between all beds, and (4) the use of well-stocked standardized dressing trolleys such that-during wound dressing changes - movements away from the bedside were minimized.

We retrospectively reviewed demographics and deep surgical culture test results of patients who developed a serious suspected SSI requiring operative management. Patients were included if they had undergone surgical exploration and had at least one intraoperative specimen sent for culture (bone, tissue, pus) and sensitivity testing.

Structured chart reviews were performed using a standardized tool. Information collected included age, gender, antimicrobial therapy, type of injury, type of initial intervention, date of diagnosis of infection, level of SSI using Centers for Disease Control (CDC) classification, surgical treatment of SSI, and total number of surgeries performed and outcome [7]. In parallel, to assure complete data, a retrospective analysis of laboratory reports from microbiological investigations of patients admitted between January 2011 and December 2012 was conducted.

The protocols of the MSF Port Harcourt surgical project were approved within the framework of a formal agreement between MSF and the Nigerian Federal Ministry of Health. The present observational study involved the analysis of routine programmatic data collected for monitoring and evaluation. All data were anonymized prior to analysis, thus satisfying the criteria for reports using routinely collected programmatic data, set by the MSF independent Ethics Review Board in Geneva, Switzerland. 


\section{Surgical interventions}

Fractures were managed according to type and location. For closed femoral shaft fractures, the SIGN (Surgical Instrument Generation Network, USA) intramedullary nailing interlocking screw system was typically employed [8]. Patients with Gustillo class I fractures underwent either internal or external fixation and Gustillo class II and III open fractures were managed with external fixation. Patients were followed after hospitalization at post-discharge days 15,45 , 90 and 180 .

The management of serious SSIs involved readmission for surgical exploration and deep tissue or bone sampling for culture and sensitivity testing, followed by targeted antimicrobial therapy. SSIs identified in this review consisted of deep infections (both CDC classification types 'deep incisional' and 'organ space' SSI) with superficial SSI excluded. Skin grafting and tissue transfer procedures were available.

\section{Laboratory testing}

During the study period, MSF collaborated with an ISO-accredited microbiology laboratory [9] and surgical specimens from patients with suspected SSI were processed according to standard microbiological methods. Antibiotic susceptibility was assessed using the Kirby-Bauer disk diffusion method, following the recommendations of the Clinical and Laboratory Standards Institute [10, 11]. The classification criteria of multidrug resistance included any of the following: Enterobacteriaceae (Escherichia coli, Klebsiella spp., Enterobacter cloacae or Proteus spp.) with resistance to a third-generation cephalosporin (cefotaxime, ceftazidime, ceftriaxone), methicillin-resistant Staphylococcus aureus (MRSA), or Pseudomonas aeruginosa with resistance to three classes of antipseudomonal antibiotics. MRSA was identified initially using cloxacillin disk diffusion and later revised to cefoxitin disk testing.

\section{Statistical analysis}

Statistical analyses were performed using $\mathrm{R}$ statistical software v. 3.0.1 (R Development Core Team, Austria). Fisher's exact tests were used to compare timing of infection (with respect to surgery, in days), in patients whose initial injury was an open fracture and with patients whose initial injury was a closed fracture requiring operative repair. $P<0.05$ was considered statistically significant.
Table 1. Characteristics of patients with surgical site infections following orthopaedic surgery in Port Harcourt, Nigeria

\begin{tabular}{ll}
\hline \hline Patient characteristic & $(N=103)$ \\
\hline Age, yr, median (IQR) & $30(24-37)$ \\
Male (\%) & 79 \\
Initial type of injury $(\%)^{*}$ & \\
$\quad$ Open fracture $\uparrow$ & 74 \\
$\quad$ Closed fracture & 18 \\
Location of injury (\%) & 54 \\
Tibia or fibula & 20 \\
Femur & 8 \\
Humerus & 5 \\
Foot & \\
Level of surgical site infection $(\%)$ & 92 \\
Organ space & 5 \\
Deep incisional & 3 \\
Unknown & \\
\hline
\end{tabular}

IQR, Interquartile range.

* Other injuries treated were degloving, lacerations and gunshot wounds.

$\dagger$ Includes open fracture types 1, 2 and 3. Patients who presented with open and closed fracture injuries $(n=4)$ were classified as open fractures.

\section{Ethical standards}

The protocols of the MSF Port Harcourt surgical project were approved within the framework of a formal agreement between MSF and the Nigerian Ministry of Health. The present observational study involved the analysis of routine programmatic data collected for monitoring and evaluation, without the use of personal identifiers, and ethical review was not sought.

\section{RESULTS}

During the study period, 120 patients were reviewed and 103 of these met the enrolment criteria. The median age was 30 years [interquartile range (IQR), 24-37] with $79 \%$ males. Seventy-four patients (72\%) were originally admitted for open fractures, requiring external fixation, and $19(18 \%)$ for closed fractures requiring open operative repair with placement of a device. The remaining injuries consisted of lacerations $(n=5)$, gunshot wounds $(n=3)$ and degloving injuries $(n=2)$. Injuries involved the tibia and/or fibula $(54 \%)$, femur $(20 \%)$, humerus $(8 \%)$ and foot $(5 \%)$ (Table 1$)$. The time between presentation to the hospital and initial operative debridement was determined in patients with open fracture $(n=74)$. For $29(39 \%)$ of 74 patients the time to initial operative procedure was 
Table 2. Management and outcomes of patients with surgical site infections in Port Harcourt, Nigeria

\begin{tabular}{ll}
\hline \hline Patient characteristic & $(N=103)$ \\
\hline Antimicrobial therapy (\%) & 43 \\
Initiated empirically & 32 \\
Initiated after culture and sensitivity report & 7 \\
No antibiotic & 18 \\
Unknown & \\
Surgical treatment of surgical site infection (\%) & 90 \\
$\quad$ Debridement & 52 \\
Skin graft & 85 \\
Amputation performed or recommended (\%) & 2 \\
No & 1 \\
Yes, performed & 12 \\
Recommended, not performed & $5(2-6)$ \\
Unknown & \\
Number of additional surgeries, median (IQR)* & $5(1$ \\
\hline
\end{tabular}

IQR, Interquartile range.

* Variable does not include original surgery that led to surgical site infection.

$<6 \mathrm{~h}$, for $19(26 \%), 6-24 \mathrm{~h}, 13(18 \%), 25-48 \mathrm{~h}, 6(8 \%)$ $>48 \mathrm{~h}$; the timing for seven patients was unknown.

Using CDC classification, $92 \%$ of infections were classified as organ space SSI and 5\% were deep incisional SSI (Table 1) [12]; debridement $(90 \%)$ and skin grafting $(52 \%)$ were the most commonly performed procedures for management of SSIs (Table 2). The median number of additional surgeries performed in Nigerian patients with SSIs was 4 (IQR 2-6), death occurred in one patient, and amputation was performed or recommended in three (Table 2).

SSI was very commonly detected more than 4 weeks after surgery; the total number of days from surgery to SSI identification was $0-14$ days $(20 \%)$, 15-28 days (21\%) and $>28$ days (58\%). Overall, 44 $(43 \%)$ of 103 SSI cases presented more than 7 weeks after surgery (Table 3). We compared the timing of SSI in patients originally presenting with open and closed fracture requiring operative repair. The median time to SSI detection for open fractures was 48.5 days (IQR 22-184.2), and 9.5 days for closed fractures (IQR 5.5-34.8) (Fisher's exact analysis: $P=0 \cdot 019$, D.F. $=4)($ Table 3).

Clinicians at Teme Hospital were permitted to initiate empirical antimicrobial therapy for suspected SSIs on clinical status of the patient prior to culture and sensitivity; therapy was initiated empirically in $43 \%$ of patients and after culture report in $32 \%$ (Table 2). Overall, 180 bacterial isolates were cultured from all 103 patients. The most common pathogens were $S$. aureus $(34 \%)$, P. aeruginosa (16\%) and E. cloacae (11\%) (Table 4) with Gram-negative organisms accounting for the majority of cases. Samples from 33 patients grew at least one MDR pathogen, including 15 with MRSA (Table 4). The resistance rates of all S. aureus isolates were $66 \%, 50 \%$ and $38 \%$ for trimethroprim-sulfamethoxazole, cloxacillin and clindamycin, respectively (Table 5). For Gram-negative pathogens, the most active agents were meropenem, imipenem, and amikacin, with resistance levels of $2 \%, 4 \%$, and $8 \%$, respectively. About one-quarter of the isolates tested were resistant to ciprofloxacin, and resistance rates for other agents was $\geqslant 35 \%$, with the exception of trimethoprim/sulfamethoxazole which was tested for only 10 isolates (Table 6).

\section{DISCUSSION}

This study has shown that deep SSIs in patients attending a West African trauma hospital had important clinical consequences, typically resulting in a need for readmission, parenteral antibiotics, additional surgical procedures, and occasionally amputation. Overall, such infections did not present as early as expected in patients with open fracture; more than half of infected open fractures presented more than 4 weeks after initial surgery. In this setting, deep SSIs were frequently caused by antibiotic-resistant bacteria, particularly Gram-negative pathogens as well as MRSA. These infections required the use of lategeneration parenteral antibiotics for definitive management, antibiotics which are not widely available in all health sectors in Nigeria.

Understanding the timing of SSIs after trauma surgery is programmatically useful for surgical programmes in low-income settings. We unexpectedly found that patients with open fractures (managed with external fixation) remain vulnerable to SSIs for a longer period of time than expected; the time to presentation was a median of nearly 50 days after surgery (Table 3 ). This is especially important because open extremity fracture is very common in this region of the world and careful, long-term patient follow-up after discharge appears critical [13]. In contrast, SSIs in patients with closed fracture undergoing internal fixation presented relatively early, a median of 9.5 days post-operatively (Table 3 ). It is worth considering that if routinely scheduled patient follow-up visits after repair of open fracture do not include the typical period for SSIs - or are poorly attended - a significant proportion of infectious complications will be missed. 
Table 3. Interval from date of surgery to infection for patients with surgical site infections in Port Harcourt, Nigeria

\begin{tabular}{llll}
\hline \hline $\begin{array}{l}\text { Timing of infection with } \\
\text { respect to surgery (days) }\end{array}$ & $\begin{array}{l}\text { Overall study } \\
\text { population }(n=103)\end{array}$ & $\begin{array}{l}\text { Open fractures } \\
(n=74)\end{array}$ & $\begin{array}{l}\text { Closed fractures } \\
(n=19)\end{array}$ \\
\hline $1-2$ weeks & 22 & 12 & 9 \\
$3-4$ weeks & 16 & 14 & 1 \\
$5-6$ weeks & 7 & 5 & 2 \\
$\geqslant 7$ weeks & 44 & 35 & 4 \\
Unknown & 14 & 8 & 3 \\
Median (IQR) & 44 days $(16-221):$ & $48 \cdot 5$ days $(22-184 \cdot 2)$ & $9 \cdot 50$ days $(5 \cdot 5-34 \cdot 8)$ \\
& & & $* P=0 \cdot 019$ \\
\hline \hline
\end{tabular}

IQR, Interquartile range.

$\dagger$ Includes open fracture types 1, 2 and 3.

$\$$ With outliers: IQR $205(n=89)$; without outliers: IQR $106 \cdot 5(n=79)$.

* Indicates significance $(P<0 \cdot 05)$. Fisher's exact test (two-sided) performed for open and closed fracture; test included unknown cells $(n=93)$.

Table 4. Frequency of bacterial species recovered from patients with surgical site infections in Port Harcourt, Nigeria

\begin{tabular}{ll}
\hline \hline Variable & $(N=103)$ \\
\hline MRSA* present (\%) & 15 \\
Multidrug resistance organism identified (\%) & 32 \\
Common pathogens (\%) & \\
S. aureus & 34 \\
$P$. aeruginosa & 16 \\
Enterobacter spp. & 19 \\
E. coli & 10 \\
\hline \hline
\end{tabular}

MRSA, Methicillin-resistant Staphylococcus aureus.

* MRSA was identified initially using cloxacillin disk diffusion and later revised to cefoxitin disk testing.

For patients with open fracture in low-income settings, given the IQR of 22-184 days, extended surveillance up to 12 months might be necessary to capture most infectious complications.

Unfortunately, achieving uniform patient follow-up can be elusive in urban West African settings where patient mobility, the high relative costs of missed workdays and transportation barriers undermine full adherence to follow-up visits. For example, the urban environment in Port Harcourt - and many other West African cities - is not easily navigated by patients with lower limb injuries and/or infection. One practical solution may be the use of phone follow-up for patients - such as those with one or more missed visits - who are at risk for becoming lost to follow-up. Standardized phone interview was shown to be a sensitive tool for detecting SSIs in a study in Great Britain. In this study, patients who endorsed - by phone interview - a possible complication were requested
Table 5. Proportion of antibiotic-resistant isolates of Staphylococcus aureus in patients with surgical site infections in Port Harcourt, Nigeria

\begin{tabular}{ll}
\hline \hline Antibiotic & \\
Resistant/tested (\%) & S. aureus \\
\hline Clindamycin & $13 / 34(38)$ \\
Erythromycin & $15 / 35(43)$ \\
Gentamicin & $12 / 32(36)$ \\
Cloxacillin & $16 / 32(50)$ \\
Fucidin & $24 / 34(71)$ \\
Penicillin & $33 / 33(100)$ \\
Cefoxitin & $3 / 4(75)$ \\
Rifampicin & $9 / 35(26)$ \\
Trimethoprim-sulfamethoxazole & $21 / 32(66)$ \\
\hline \hline
\end{tabular}

to present for complete physical examination evaluation [14]. An internal exercise by MSF in Nigeria showed that a significant proportion of orthopaedic trauma patients did not attend follow-up visits through 180 days but that most patients lost to follow-up could be located with cell phone outreach [15].

A clearer understanding of pathways to reduce SSIs in West African settings is important. Although more research is needed, a few important studies do exist; a report from Kenya suggested that merely increasing the use of standard perioperative antibiotic prophylaxis (and discouraging extended post-operative use of antibiotics) had a demonstrable impact on reducing SSIs and a second study in obstetric patients in sub-Saharan Africa also suggested no advantage to extended antibiotic prophylaxis [16, 17]. Initial steps to reduce SSIs in West Africa might be profitably focused on improving uptake of existing interventions - including increasing 
Table 6. Proportion of antibiotic-resistant isolates of Gram-negative pathogens isolated from patients with surgical site infections in Port Harcourt, Nigeria

\begin{tabular}{lllll}
\hline \hline Antibiotic & $\begin{array}{l}\text { E. cloacae } \\
(N=11)\end{array}$ & $\begin{array}{l}\text { E. coli } \\
(N=10)\end{array}$ & $\begin{array}{l}\text { E. sakazakii } \\
(N=8)\end{array}$ & $\begin{array}{l}\text { P. aeruginosa } \\
(N=16)\end{array}$ \\
\hline Resistant/tested (\%) & $4 / 10(40)$ & $5 / 9(56)$ & $4 / 8(50)$ & $3 / 16(19)$ \\
Gentamicin & $8 / 10(80)$ & $6 / 10(60)$ & $8 / 8(100)$ & - \\
Cefoxitin & $3 / 5(60)$ & $4 / 5(80)$ & - & - \\
Trimethoprim-sulfamethoxazole & $2 / 11(18)$ & $2 / 10(20)$ & $0 / 8(0)$ & $0 / 16(0)$ \\
Amikacin & $6 / 11(55)$ & $6 / 10(60)$ & $6 / 7(86)$ & $1 / 16(6)$ \\
Cefepime & $6 / 11(55)$ & $6 / 10(60)$ & $7 / 8(88)$ & $1 / 16(6)$ \\
Ceftazidime & $3 / 11(27)$ & $4 / 10(40)$ & $3 / 8(38)$ & $1 / 16(6)$ \\
Ciprofloxacin & $0 / 11(0)$ & $1 / 10(10)$ & $1 / 8(13)$ & $0 / 16(0)$ \\
Imipenem & $0 / 10(0)$ & $1 / 10(10)$ & $0 / 8(0)$ & $0 / 16(0)$ \\
Meropenem & $6 / 10(60)$ & $6 / 10(60)$ & $6 / 8(75)$ & $1 / 16(6)$ \\
Piperacillin/tazobactam & $6 / 10(60)$ & $7 / 9(78)$ & $3 / 7(43)$ & $0 / 16(0)$ \\
Tobramycin & $10 / 10(100)$ & $10 / 10(100)$ & $8 / 8(100)$ & - \\
Ampicillin & $10 / 10(100)$ & $8 / 10(80)$ & $8 / 8(100$ & - \\
Amoxicillin/clavulanate & $7 / 10(70)$ & $6 / 10(60)$ & $7 / 8(88)$ & - \\
Cefotaxime & $7 / 10(70)$ & $6 / 10(60)$ & $7 / 8(88)$ & - \\
Ceftriaxone & $10 / 10(100)$ & $7 / 10(70)$ & $7 / 8(88)$ & - \\
Cefuroxime &
\end{tabular}

- , Denotes not tested.

use of single-dose perioperative antibiotic prophylaxis/ eliminating extended prophylaxis, improving nurseto-patient ratios, reducing crowding and improving rates of hand hygiene - already known to be effective but not well implemented. One challenge is that the visibility of infection prevention activities in hospitals is not yet high in this region. However, the recent emergence of Ebola virus disease has brought attention to the need to assure safer hospital patient care in West Africa. This attention might be leveraged to assure that every hospital has essential ingredients in place to implement and monitor infection prevention activities including infection prevention committees, staff training, dedicated human resources and essential materials. Expanded prevention activities will need adequate funding and support from central governments and donors.

MDR pathogens appear to be of growing importance in SSIs in West Africa [18]. Among other implications, this situation should bring attention to the need to improve availability of high-quality microbiological facilities in support of surgical programmes. At Teme Hospital, a model was adopted involving the use of air transport several times per week to link the hospital with such a laboratory in the capital Lagos. The use of internet-based reporting reduced the time between the finalization of culture results and initiation of organism-specific antibiotic therapy. However, such linkages remain uncommon in West
Africa and are also novel for humanitarian surgical programmes. The quality of microbiology available in support of surgery is poor and therefore the epidemiology of SSIs - or other common infections - in individual hospitals cannot be established. As a result, for individual patients with a SSI, the initial as well as definitive antibiotics are often empirical in contrast to the more optimal strategy involving a broad-spectrum first-line regimen (based on institution-specific patterns in similar patients), given briefly followed by definitive therapy, based on patient-specific, intraoperative cultures available within 3-7 days.

In contexts where MDR bacterial pathogens are prevalent - and Gram-negative pathogens are implicated in a greater proportion of infections - the choice of antimicrobial is difficult. Greater access to microbiology will also help make clear which late-generation antibiotics are needed for surgical programmes in this part of the world. Currently, key classes of antibiotics including glycopeptides, carbapenems and extendedspectrum aminoglycosides are not widely available in West Africa, and it is difficult to build a strong argument to prioritize access to these agents without more microbiology capacity to provide the relevant epidemiology.

This study has several limitations. First, as a result of our study design we were not able to estimate the global rate of SSIs after orthopaedic trauma surgery. Patients at Teme Hospital with superficial SSIs were 
not evaluated in the operating room and did not routinely undergo culture and sensitivity testing. Other studies have attempted to define the global SSI rate, including superficial infections, in the West African setting, as noted earlier [2, 19]. Second, we were not able to describe specific risk factors for SSIs because patients without a SSI were not similarly investigated. Future research might prospectively follow patients including those with open fracture - at high risk for SSI to determine the critical risk factors to focus better surveillance and preventive efforts. Despite these limitations, our results show that deep SSI was a common complication of orthopaedic trauma surgery, particularly after open fracture and we were able to establish timing and microbiological data more precisely than in prior published studies.

In conclusion, deep SSIs in a medical humanitarian trauma surgical project commonly occurred more than 4 weeks after initial surgery, suggesting the need for active monitoring of patients after discharge particularly after open fracture. The association of MDR organisms with such infections has serious implications, often resulting in the need for readmission for multiple surgical procedures, frequently requiring late-generation antibiotics, and in worst cases, amputation. The appropriate management of SSIs in West Africa will require broader access to surgical and microbiology services, active patient follow-up and wider availability of appropriate parenteral antibiotics for patients with confirmed MDR infections.

\section{ACKNOWLEDGEMENTS}

We acknowledge the essential work of partners at Teme Hospital including Samuel Okere (laboratory) and the tremendous efforts of the cleaning staff, nurses, and physicians to reduce hospital-acquired infection. No outside funding was used in the production of this manuscript

\section{DECLARATION OF INTEREST}

None.

\section{REFERENCES}

1. Allegranzi B, et al. Burden of endemic health-care-associated infection in developing countries: systematic review and meta-analysis. Lancet 2010; 377: 228-241.

2. Nthumba PM, et al. Cluster-randomized, crossover trial of the efficacy of plain soap and water versus alcohol- based rub for surgical hand preparation in a rural hospital in Kenya. British Journal of Surgery 2010; 97: 1621-1628.

3. Saxer F, et al. Benefit of a single preoperative dose of antibiotics in a sub-saharan district hospital: minimal input, massive impact. Annals of Surgery 2009; 249: 322-326.

4. Médecins Sans Frontières (MSF). International Activity Report 2011, pp. 82-83 (http://www.msf.org/en/msfinternational-activity-report-2011).

5. Médecins Sans Frontières (MSF). International Activity Report 2012, pp. 72-73.

6. Médecins Sans Frontières (MSF). Operations Analysis MSF International Typology, 2011, pp. 155-166.

7. Horan TC, Andrus M, Dudeck MA. CDC/NHSN surveillance definition of health care-associated infection and criteria for specific types of infections in the acute care setting. American Journal of Infection Control 2008; 36: 309-332.

8. Zirkle L Jr., Injuries in developing countries - how can we help?: The role of orthopaedic surgeons. Clinical Orthopaedics \& Related Research 2008; 466: 2443-2450.

9. Pathcare. 2013 (http: //www.pathcarenigeria.com/).

10. Clinical and Laboratory Standards Institute (CLSI). Performance standards for antimicrobial disk susceptibility tests; Approved Standard, 11th edn. CLSI document M02-A11. Wayne, PA: Clinical and Laboratory Standards Institute, 2012.

11. Clinical and Laboratory Standards Institute (CLSI). Performance standards for antimicrobial susceptibility testing; Twenty-Second Informational Supplement. CLSI document M100-S22. Wayne, PA: Clinical and Laboratory Standards Institute, 2012.

12. Mangram A, et al. The Hospital Infection Control Practices Advisory Committee. Guideline for prevention of surgical site infection, 1999. Infection Control and Hospital Epidemiology 1999; 20: 250-280.

13. Ikem I, Oginni L, Bamgboye E. Open fractures of the lower limb in Nigeria. International Orthopaedics (SICOT) 2001; 25: 386-388.

14. Taylor EW, et al. Telephone call contact for postdischarge surveillance of surgical site infections. A pilot, methodological study. Journal of Hospital Infection 2003; 55: 8-13.

15. Médecins Sans Frontières (MSF). Internal data, 2011.

16. Aiken AM, et al. Changing sse of surgical antibiotic prophylaxis in Thika Hospital, Kenya: A quality improvement intervention with an interrupted time series design. PLoS ONE 2013; 8: 1-6.

17. Westen E, et al. Single-dose compared with multiple day antibiotic prophylaxis for cesarean section in lowresource settings, a randomized controlled, noninferiority trial. Acta Obstetricia et Gynecologica Scandinavica 2015; 94: 43-49.

18. Scherbaum M, et al. Incidence, pathogens and resistance patterns of nosocomial infections at a rural hospital in Gabon. BMC Infectious Diseases 2014; 14: 1-15.

19. Nwankwo E, Edino S. Seasonal variation and risk factors associated with surgical site infection rate in Kano, Nigeria. Turkish Journal of Medical Sciences 2014; 44: 674-680. 